\title{
Impact of different training modalities on glycaemic control and blood lipids in patients with type 2 diabetes: a systematic review and network meta-analysis
}

\author{
Lukas Schwingshackl • Benjamin Missbach • Sofia Dias • \\ Jürgen König • Georg Hoffmann
}

Received: 13 March 2014 / Accepted: 2 June 2014 / Published online: 5 July 2014

(C) Springer-Verlag Berlin Heidelberg 2014

\begin{abstract}
Aims/hypothesis This study aimed to systematically review randomised controlled trials comparing the effects of aerobic exercise training (AET), resistance training (RT) and combined training (CT) on glycaemic control and blood lipids in patients with type 2 diabetes mellitus.

Methods Searches were performed in MEDLINE, EMBASE and the Cochrane Library. Inclusion criteria were: type 2 diabetes mellitus, adult, supervised training and a minimum intervention period of 8 weeks. Pooled effects were calculated by fixed/random effect pairwise and Bayesian fixed/random effects network meta-analyses.

Results A total of 14 trials enrolling 915 participants were included. AET was more effective than RT in improving $\mathrm{HbA}_{1 \mathrm{c}}$ levels (mean difference [MD] $-0.20 \%$ $[-2.2 \mathrm{mmol} / \mathrm{mol}] ; 95 \%$ CI $-0.32,-0.08 ; p=0.0007$, 10 trials $/ 515$ participants) and fasting glucose (MD $-0.9 \mathrm{mmol} / 1 ; 95 \% \mathrm{CI}-1.71,-0.09 ; p=0.03,8$ trials $/ 245$ participants). Compared with AET, CT resulted in a significantly more pronounced reduction in $\mathrm{HbA}_{1 \mathrm{c}}(\mathrm{MD}-0.17 \%$ $[-1.87 \mathrm{mmol} / \mathrm{mol}] ; 95 \% \mathrm{CI}-0.31,-0.03 ; p=0.02,9$ trials $/ 493$ participants). Compared with RT, the MD of the change in $\mathrm{HbA}_{1 \mathrm{c}}(\mathrm{MD}-0.62 \%,[-6.82 \mathrm{mmol} / \mathrm{mol}] ; 95 \% \mathrm{CI}-0.95$,
\end{abstract}

Electronic supplementary material The online version of this article (doi:10.1007/s00125-014-3303-z) contains peer-reviewed but unedited supplementary material, which is available to authorised users.

L. Schwingshackl $(\bowtie) \cdot$ B. Missbach $\cdot$ J. König $\cdot$ G. Hoffmann Department of Nutritional Sciences, Faculty of Life Sciences, University of Vienna, Althanstraße 14 (UZAII), 1090 Vienna, Austria

e-mail: lukas.schwingshackl@univie.ac.at

S. Dias

School of Social and Community Medicine, University of Bristol, Bristol, UK
$-0.30 ; p=0.0002,5$ trials $/ 362$ participants], fasting glucose (MD $-1.99 \mathrm{mmol} / \mathrm{l} ; 95 \% \mathrm{CI}-3.07,-0.90 ; p=0.0003,3$ trials/ 99 participants) and triacylglycerols (MD $-0.28 \mathrm{mmol} / \mathrm{l} ; 95 \%$ CI $-0.46,-0.10 ; p=0.003,4$ trials $/ 213$ participants) were all in favour of CT. The exclusion of trials with a high risk of bias yielded only non-significant results.

Conclusions/interpretation The present data suggest that CT might be the most efficacious exercise modality to improve glycaemic control and blood lipids. Interpretation with respect to clinical relevance is limited by the low quality of the studies included and the limited information on the clinically important outcomes or adverse effects of exercise.

Keywords Aerobic exercise $\cdot$ Combined training $\cdot$ Network meta-analysis $\cdot$ Resistance training $\cdot$ Systematic review

\begin{tabular}{ll}
\multicolumn{2}{l}{ Abbreviations } \\
AET & Aerobic exercise training \\
BW & Body weight \\
CT & Combined training \\
DBP & Diastolic blood pressure \\
FG & Fasting glucose \\
MD & Mean difference \\
RT & Resistance training \\
SBP & Systolic blood pressure \\
TC & Total cholesterol \\
TG & Triacylglycerols
\end{tabular}

Introduction

Increased physical activity and improved nutritional habits in the form of hypocaloric diets (of varying macronutrient compositions) are of particular importance to decelerate the manifestations of type 2 diabetes [1-3]. The ADA and the 
American College of Sports Medicine have stated that a combination of resistance training (RT) and aerobic exercise training (AET) of at least $150 \mathrm{~min}$ of moderate-intensity exercise per week may be more effective in improving glycaemic control than focusing solely on one single training modality (evidence category B) [4].

The isolated effects of either RT or AET, or a combination of both (combined training [CT]), on anthropometric, cardiac and metabolic risk factors have been meta-analysed by Snowling and Hopkins [5] as well as by Chudyk and Petrella [6]. Both studies reported that the reduction in $\mathrm{HbA}_{1 \mathrm{c}}$ and fasting glucose (FG) levels, systolic blood pressure (SBP), waist circumference, HDL and triacylglycerols (TG) was more pronounced following AET and CT compared with RT. In addition, $\mathrm{HbA}_{1 \mathrm{c}^{-}}$and blood pressure-lowering effects of RT were shown. However, all these systematic reviews included trials in which training modalities were compared with the data from a sedentary control group [7,8].

To date, no systematic review has compared the direct and indirect effects of these three different training modalities on the outcomes of glycaemic control and blood lipids in patients with type 2 diabetes. A recent pairwise meta-analysis comparing RT (all supervised) with AET (not all supervised) exercise in patients with type 2 diabetes concluded that although differences in some outcome variables reached statistical significance, there was no evidence that they were of clinical relevance [9]. In a recently published network metaanalysis, we were able to demonstrate that $\mathrm{CT}$ is ranked as the most likely effective exercise model in the treatment of overweight and obesity [10].

The aim of the present study was to assess the efficacy of AET, RT and CT on glycaemic control, blood pressure and blood lipids in patients with type 2 diabetes mellitus in a systematic review including a pairwise and network metaanalysis of randomised trials. The importance of supervised training has been demonstrated by Umpierre et al, who showed that structured training compared with training advice only significantly improved glycaemic control in patients with type 2 diabetes [11]. Based upon these findings, only trials that conducted an exercise intervention which was guided by supervised training were enrolled in the present systematic review and meta-analyses.

\section{Methods}

The review was registered in PROSPERO International Prospective Register of Systematic Reviews (www.crd.york. ac.uk/prospero/index.asp, identifier CRD42014007502). However, no study protocol was published before the initiation of the meta-analysis.
Literature search

Queries of the literature were performed using the electronic databases MEDLINE (until 2 May 2014), EMBASE (until 2 May 2014) and the Cochrane Central Register of Controlled Trials (until 2 May 2014) with no restrictions. The following keywords were used: ('strength' OR 'resistance' OR 'aerobic' OR 'endurance' OR 'combined training' OR 'progressive' OR 'walking' OR 'interval training' OR 'weight lifting') AND ('training' OR 'exercise' OR 'physical activity') AND ('diabetes' OR 'glycemic' OR 'glycaemia' OR 'glycaemic' OR 'glycemia' OR ' $\mathrm{HbA}_{1 \mathrm{c}}$ ' OR ' $\mathrm{A}_{1 \mathrm{c}}$ ' OR 'glycated' $\mathrm{OR}$ 'glycosylated' OR 'glucose' OR 'lipids' OR 'body weight' OR 'blood pressure') AND ('randomized controlled trial' OR 'randomized' OR 'clinical trials as topic' OR 'placebo' OR 'randomly' OR 'trial') NOT ('animals' NOT 'humans').

Moreover, the reference lists from the retrieved articles, systematic reviews and meta-analyses were checked to search for further relevant studies. This systematic review was planned, conducted and reported in adherence with standards of quality for reporting meta-analyses [12]. The entire literature search was conducted independently by two authors (L. Schwingshackl and B. Missbach), with disagreements resolved by consensus. The detailed search strategy for MEDLINE is given in the electronic supplementary material (ESM Methods).

\section{Eligibility criteria}

Studies were included in the meta-analysis if they met all of the following criteria: (1) a randomised controlled design; (2) a minimum intervention period of 8 weeks; (3) patients with type 2 diabetes; (4) patients' age $\geq 19$ years; (5) a comparison of either AET vs RT and/or CT vs AET and/or CT vs RT; (6) an assessment of at least one of the following outcome markers: $\mathrm{HbA}_{1 \mathrm{c}}$, blood glucose, body weight (BW), blood pressure or blood lipids (total cholesterol [TC], LDL, HDL and TG); (7) the reporting of changes from baseline value scores with SDs (or data suitable to calculate these variables: $\mathrm{SE}$ and $95 \% \mathrm{CI}$ ); if the SDs of the changes from baseline value scores were not available, post-intervention values were imputed, according to the Cochrane Handbook [13]; (8) training that was conducted under direct (guided by a physiotherapist in training classes, hospital gyms, etc.) or partial supervision, and was not home-based; and (9) the exclusion of studies with a dietary co-intervention that was not applied in all the intervention groups. All abstracts and full texts were independently assessed for eligibility by two authors (L. Schwingshackl and B. Missbach).

Risk of bias assessment

Full copies of the studies were independently assessed by two authors (L. Schwingshackl and B. Missbach) for 
methodological quality using the risk of bias assessment tool from the Cochrane Collaboration [13, 14]. The following sources of bias were detected: selection bias (random sequence generation and allocation concealment), detection bias (blinding of outcome assessment), blinding of participants and personnel (performance bias), attrition bias (incomplete outcome data) and reporting bias (selective reporting) (ESM Fig. 1).

\section{Data extraction and statistical analysis}

The following data were extracted from each study: the first author's last name, publication year, study duration, participant's sex, age and BMI, sample size, duration of diabetes, $\mathrm{HbA}_{1 \mathrm{c}}$ at baseline, drug treatment, change of treatment during the trial, treatment effects, intervention type, dose, intensity and frequency, and differences in the means of two time points or post-intervention mean values with corresponding SDs. For each outcome measure of interest, pairwise and network random effects meta-analyses were performed in order to determine the pooled relative effect of each intervention relative to every other intervention in terms of the mean differences (MDs) between the changes from baseline value scores (or post-intervention values) of the different interventions. To process the data for the meta-analysis, we imputed the data for the changes from baseline means and their SDs. When the SDs for the changes from baseline values were not available [15-20], the postintervention values with the corresponding SDs were imputed, according the guidelines of the Cochrane Handbook [13].

Data were pooled if outcomes were reported by at least three studies. Heterogeneity between trial results was tested with a Cochran's Q test. A value for $I^{2}$ of $>50 \%$ was considered to represent substantial heterogeneity [21]. When substantial heterogeneity was present, the random effects model was used to estimate MDs with $95 \%$ CIs. Forest plots were generated to illustrate the study-specific effect sizes along with a $95 \%$ CI. To determine the presence of publication bias, the symmetry of the funnel plots in which mean MDs were plotted against their corresponding SEs were assessed. Additionally, Begg's and Egger's regression tests were performed to detect small study effects [22, 23].

Separate pairwise meta-analyses were first used to compare all the interventions. Network meta-analysis was then used to synthesise all the available evidence [24]. Network metaanalysis methods are extensions of the standard pairwise meta-analysis model that enable a simultaneous comparison of multiple interventions while preserving the internal randomisation of individual trials. They have the advantage of adequately accounting for the correlation in relative effect estimates from three-arm trials as well as providing a single coherent summary of all the evidence. Random effects network meta-analysis models were used when substantial heterogeneity was found in any of the pairwise comparisons for that outcome. Otherwise, the choice between fixed and random effects was made by comparing the deviance information criteria for each model $[24,25]$. The model with the lowest deviance information criterion was chosen (differences $>3$ are considered meaningful). Pooled effect sizes from the network meta-analyses are presented as posterior medians and $95 \%$ credible intervals (i.e. the Bayesian equivalent of CIs) in the appropriate units, along with the estimated between-study heterogeneity.

For pairwise meta-analyses, data were analysed using Review Manager 5.1 software, provided by the Cochrane Collaboration (http://ims.Cochrane.org/revman). Network meta-analyses were conducted using Markov chain Monte Carlo simulation implemented with the open-source software WinBUGS, version 1.4.3 [26]. The WinBUGS code used is freely available online $[24,27]$ (program 'TSD2-5aRE Normal_id.odc' or 'TSD2-5aFE_Normal_id.odc').

Minimally informative normal priors were used for all treatment effect variables and a uniform prior $(0,150)$ was used for the between-study SD (heterogeneity) variable. Sensitivity to this prior was assessed, but there was no meaningful change in the relative effects or overall conclusions.

Three Markov chain Monte Carlo chains were used to assess convergence using Brooks-Gelman-Rubin plots and inspection of the trace plots [28]. Convergence was achieved after 20,000 iterations for all outcomes. Posterior summaries were then obtained from a further simulation of 50,000 iterations in each of the three chains (giving 150,000 in total), resulting in a small Monte Carlo error.

The potential for inconsistency was assessed by inspection of the available evidence. In case of possible inconsistency, Bayesian $p$ values for the difference between direct and indirect evidence were calculated, and direct and indirect estimates were compared [29, 30].

\section{Results}

Overall, a total of 14 trials (16 reports) extracted from 9,477 articles met the eligibility requirements and were included for the present systematic review and meta-analysis [15-20, 31-40]. One study was excluded since it was not described as randomised [41], and two trials provided no information on whether the AET was supervised $[42,43]$. The detailed steps of the article selection process for the meta-analysis are described as a flow diagram in ESM Fig. 2. The studies were published between 2003 and 2013 and had enrolled a total of 915 participants. The study duration ranged between 2 and 12 months; the patients' mean age was between 49 and 62.5 years, and their BMI between 27.1 and $43.8 \mathrm{~kg} / \mathrm{m}^{2}$. Fourteen trials met the objectives for meta-analysis: $10 \mathrm{com}$ pared RT vs AET, 9 compared CT vs AET, and 5 compared CT vs RT (ESM Fig. 3). The general and specific study 
characteristics are summarised in Table 1, ESM Table 1 and ESM Table 2.

The pairwise pooled estimate of effect size for the effects of RT vs AET, CT vs AET and CT vs RT on glycaemic control, blood pressure and blood lipids are summarised in Table 2.

Pairwise meta-analysis

Primary outcome The reduction in $\mathrm{HbA}_{1 \mathrm{c}}$ (MD $-0.20 \%$ $[-2.2 \mathrm{mmol} / \mathrm{mol}] ; 95 \%$ CI $-0.32,-0.08 ; p=0.0007$, $I^{2}=26 \%$, 10 trials, 515 participants) (ESM Fig. 4) was significantly more pronounced in the AET groups compared with the RT groups. When compared with AET and $\mathrm{RT}$, the $\mathrm{CT}$ protocols resulted in a significant reduction in $\mathrm{HbA}_{1 \mathrm{c}}(\mathrm{MD}-0.17 \%$ [-1.87 $\mathrm{mmol} / \mathrm{mol}]$; $95 \%$ CI -0.31 to $-0.03 ; p=0.02, I^{2}=21 \%, 9$ trials, 493 participants) (ESM Fig. 5) and (MD $-0.62 \%$, $[-6.82 \mathrm{mmol} / \mathrm{mol}] ; 95 \%$ CI $-0.95,-0.30 ; p=0.0002$, $I^{2}=74 \%$, 5 trials, 362 participants) (ESM Fig. 6).

Secondary outcomes No significant differences were observed for BW, diastolic blood pressure (DBP), SBP, TC,

Table 1 General study characteristics

\begin{tabular}{|c|c|c|c|c|c|c|}
\hline Reference & $\begin{array}{l}\text { Sample size, } \\
\text { mean baseline } \\
\text { BMI }\left(\mathrm{kg} / \mathrm{m}^{2}\right)\end{array}$ & $\begin{array}{l}\text { Mean age } \\
\text { (years), } \\
\text { female }(\%)\end{array}$ & $\begin{array}{l}\text { Study } \\
\text { duration } \\
\text { (months) }\end{array}$ & Comparisons & $\begin{array}{l}\text { Timing of post-intervention } \\
\text { measurement since last } \\
\text { exercise session }\end{array}$ & Findings \\
\hline Bacchi et al 2012 [31] & $\begin{array}{l}40 \\
29.35\end{array}$ & $\begin{array}{l}56.4 \\
30\end{array}$ & 4 & $\begin{array}{l}\text { RT vs } \\
\text { AET }\end{array}$ & n.d. & $\begin{array}{l}\mathrm{RT}: / \\
\mathrm{AET}: \uparrow \uparrow \dot{V} \mathrm{O}_{2 \max }\end{array}$ \\
\hline $\begin{array}{c}\text { Balducci et al } \\
2010[17]\end{array}$ & $\begin{array}{l}42 \\
30\end{array}$ & $\begin{array}{l}62.5 \\
38\end{array}$ & 12 & $\begin{array}{l}\text { AET vs } \\
\text { CT }\end{array}$ & n.d. & $\begin{array}{l}\mathrm{AET}: \downarrow \mathrm{WC} ; \uparrow \dot{V} \mathrm{O}_{2 \max }, \mathrm{HDL} \\
\mathrm{CT}: \downarrow \mathrm{BW}, \mathrm{WC} ; \uparrow \dot{V} \mathrm{O}_{2 \max }, \mathrm{HDL}\end{array}$ \\
\hline $\begin{array}{r}\text { Church et al } \\
2010[32]\end{array}$ & $\begin{array}{l}221 \\
34.9\end{array}$ & $\begin{array}{l}55.3 \\
62\end{array}$ & 9 & $\begin{array}{l}\text { RT vs } \\
\text { AET vs } \\
\text { CT }\end{array}$ & $\begin{array}{l}48-72 \mathrm{~h} \text { after last } \\
\text { exercise test }\end{array}$ & $\begin{array}{l}\mathrm{RT}: \downarrow \mathrm{FM} \\
\mathrm{AET}: \downarrow \mathrm{LBM} \\
\mathrm{CT}: \downarrow \mathrm{BW}, \mathrm{FM}, \uparrow \dot{V} \mathrm{O}_{2 \max }\end{array}$ \\
\hline Cuff et al 2003 [33] & $\begin{array}{l}19 \\
32.9\end{array}$ & $\begin{array}{l}61.4 \\
100\end{array}$ & 4 & $\begin{array}{l}\text { AET vs } \\
\text { CT }\end{array}$ & n.d. & $\begin{array}{l}\mathrm{AET}: \downarrow \downarrow \mathrm{BW} \\
\mathrm{CT}: \downarrow \downarrow \mathrm{BW}\end{array}$ \\
\hline Gram et al 2010 [19] & $\begin{array}{l}68 \\
32.3\end{array}$ & $\begin{array}{l}60.6 \\
46\end{array}$ & 4 & $\begin{array}{l}\text { AET vs } \\
\text { CT }\end{array}$ & n.d. & $\begin{array}{l}\text { AET: / } \\
\text { CT: LDL } \downarrow \downarrow\end{array}$ \\
\hline $\begin{array}{l}\text { Jorge et al } 2011[15] \\
\text { de Oliveira et al } \\
2012[20]\end{array}$ & $\begin{array}{l}36 \\
30.6\end{array}$ & $\begin{array}{l}54.7 \\
61\end{array}$ & 3 & $\begin{array}{l}\text { RT vs } \\
\text { AET vs } \\
\text { CT }\end{array}$ & n.d. & $\begin{array}{l}\text { RT: } \downarrow \text { TC, HDL, TG } \\
\text { AET: } \downarrow \text { TC, HDL, TG, } \uparrow \dot{V} \mathrm{O}_{2 \max } \\
\text { CT: } \downarrow \text { TC, TG }\end{array}$ \\
\hline $\begin{array}{l}\text { Kwon et al } 2011[34] \\
\text { Ku et al } 2010[35]\end{array}$ & $\begin{array}{l}28 \\
27.1\end{array}$ & $\begin{array}{l}55.9 \\
100\end{array}$ & 3 & $\begin{array}{l}\text { RT vs } \\
\text { AET }\end{array}$ & n.d. & $\begin{array}{l}\mathrm{RT}: \downarrow \mathrm{BW} \\
\mathrm{AET}: \downarrow \mathrm{BW}, \uparrow \dot{V} \mathrm{O}_{2 \max }\end{array}$ \\
\hline $\begin{array}{l}\text { Kadoglou et al } \\
2013 \text { [36] }\end{array}$ & $\begin{array}{l}66 \\
32.1\end{array}$ & $\begin{array}{l}57.4 \\
27\end{array}$ & 6 & $\begin{array}{l}\text { RT vs } \\
\text { AET vs } \\
\text { CT }\end{array}$ & n.d. & $\begin{array}{l}\text { AET: } \downarrow \text { SBP, FG, } \mathrm{HbA}_{1 \mathrm{c}}, \mathrm{HOMA}-\mathrm{IR}, \\
\text { FI, TC, TG; } \uparrow \dot{V} \mathrm{O}_{2 \max } \\
\text { RT: } \downarrow \text { SBP, FG, } \mathrm{HbA}_{1 \mathrm{c}}, \mathrm{TC}, \mathrm{TG}, \\
\text { HOMR-IR, FI } \\
\text { CT: } \downarrow \text { SBP, FG, HbA } \mathrm{Hb}_{1 \mathrm{c}}, \mathrm{HOMA}-\mathrm{IR}, \\
\text { FI, TC, TG, FM } \uparrow \dot{V} \mathrm{O}_{2 \max }\end{array}$ \\
\hline $\begin{array}{l}\text { Lambers et al } \\
2008[18]\end{array}$ & $\begin{array}{l}35 \\
29.8\end{array}$ & $\begin{array}{l}54 \\
34\end{array}$ & 3 & $\begin{array}{l}\text { AET vs } \\
\text { CT }\end{array}$ & $\begin{array}{l}\text { After last exercise and } \\
\text { overnight fasting }\end{array}$ & $\begin{array}{l}\text { AET: / } \\
\text { CT: } \downarrow \downarrow \mathrm{HbA}_{1 \mathrm{c}}, \downarrow \downarrow \mathrm{TC}\end{array}$ \\
\hline Moe et al 2011 [37] & $\begin{array}{l}26 \\
30\end{array}$ & $\begin{array}{l}57 \\
0\end{array}$ & 3 & $\begin{array}{l}\text { RT vs } \\
\text { AET }\end{array}$ & $\begin{array}{l}48 \mathrm{~h} \text { after last exercise } \\
\text { tests }\end{array}$ & $\begin{array}{l}\mathrm{AET}: \downarrow \mathrm{HbA}_{1 \mathrm{c}} ; \uparrow \dot{V} \mathrm{O}_{2 \max } \\
\mathrm{RT}: \downarrow \mathrm{WHR} \mathrm{HbA}_{1 \mathrm{c}}\end{array}$ \\
\hline $\mathrm{Ng}$ et al 2010 [38] & $\begin{array}{l}60 \\
27.6\end{array}$ & $\begin{array}{l}58 \\
68\end{array}$ & 2 & $\begin{array}{l}\text { RT vs } \\
\text { AET }\end{array}$ & n.d. & $\begin{array}{l}\mathrm{RT}: \downarrow \downarrow \mathrm{WC} \\
\mathrm{AET}: \uparrow \uparrow \dot{V} \mathrm{O}_{2 \max }\end{array}$ \\
\hline Sigal et al 2007 [16] & $\begin{array}{l}188 \\
34.9\end{array}$ & $\begin{array}{l}54 \\
63\end{array}$ & 6 & $\begin{array}{l}\text { RT vs } \\
\text { AET vs } \\
\text { CT }\end{array}$ & n.d. & $\begin{array}{l}\mathrm{RT}: \downarrow \downarrow \mathrm{HbA}_{1 \mathrm{c}} \\
\mathrm{AET}: \downarrow \downarrow \mathrm{HbA}_{1 \mathrm{c}}, \mathrm{BW}, \mathrm{WC}, \mathrm{FM} \\
\mathrm{CT}: \downarrow \downarrow \mathrm{HbA}_{1 \mathrm{c}}\end{array}$ \\
\hline Sukala et al 2012 [39] & $\begin{array}{l}26 \\
43.8\end{array}$ & $\begin{array}{l}49 \\
72\end{array}$ & 4 & $\begin{array}{l}\text { RT vs } \\
\text { AET }\end{array}$ & $\begin{array}{l}72 \mathrm{~h} \text { after last exercise } \\
\text { tests }\end{array}$ & $\begin{array}{l}\text { RT: / } \\
\text { AET: } \downarrow \text { TG }\end{array}$ \\
\hline Yavari et al 2012 [40] & $\begin{array}{l}60 \\
29.5\end{array}$ & $\begin{array}{l}50.2 \\
\text { n.d } \\
\text { n.d }\end{array}$ & 12 & $\begin{array}{l}\text { RT vs } \\
\text { AET vs } \\
\text { CT }\end{array}$ & n.d. & $\begin{array}{l}\mathrm{AET}: \downarrow \mathrm{HbA}_{1 \mathrm{c}}, \mathrm{FG}, \mathrm{TG} \uparrow \dot{V} \mathrm{O}_{2 \max } \\
\mathrm{RT}: \downarrow \mathrm{HbA}_{1 \mathrm{c}}, \mathrm{FG}, \mathrm{FM}, \uparrow \dot{V} \mathrm{O}_{2 \max } \\
\mathrm{CT}: \downarrow \mathrm{HbA}_{1 \mathrm{c}}, \mathrm{FG}, \mathrm{BMI}, \mathrm{FM} \uparrow \dot{V} \mathrm{O}_{2 \max }\end{array}$ \\
\hline
\end{tabular}

/, no significant within/between-group changes; $\downarrow \downarrow$, $\uparrow \uparrow$ significant between-group changes (decreases and increases, respectively; including control group comparison); $\downarrow$, $\uparrow$ significant within-group changes (decreases and increases, respectively)

n.d., no data; $\dot{V} \mathrm{O}_{2 \max }$, maximal oxygen uptake; WC, waist circumference; FM, fat mass; LBM, lean body mass; FI, fasting insulin 
Table 2 Pooled estimates (pairwise fixed or random effect meta-analysis) of effect size (95\% CIs) expressed as MD for the effects of AET vs RT, CT vs AET and CT vs RT on glycaemic control, blood lipids, blood pressure and BW

\begin{tabular}{|c|c|c|c|c|c|c|c|}
\hline Outcomes & No. of studies & Sample size & $\mathrm{MD}^{\mathrm{a}}$ & $95 \% \mathrm{CI}$ & $p$ values & Inconsistency $I^{2}$ & Egger test \\
\hline \multicolumn{8}{|l|}{ AET vs RT } \\
\hline $\mathrm{HbA}_{1 \mathrm{c}}(\%)$ & 10 & 515 & -0.20 & $-0.32,-0.08$ & 0.0007 & $26 \%$ & 0.80 \\
\hline $\mathrm{HbA}_{1 \mathrm{c}}(\mathrm{mmol} / \mathrm{mol})$ & & & -2.20 & $-3.52,-0.88$ & & & \\
\hline $\mathrm{FG}(\mathrm{mmol} / \mathrm{l})$ & 8 & 245 & -0.90 & $-1.71,-0.09$ & 0.03 & $72 \%$ & 0.66 \\
\hline $\mathrm{TC}(\mathrm{mmol} / \mathrm{l})$ & 8 & 262 & 0.06 & $-0.11,0.22$ & 0.50 & $26 \%$ & 0.64 \\
\hline LDL (mmol/l) & 9 & 372 & -0.03 & $-0.15,0.09$ & 0.62 & $32 \%$ & 0.28 \\
\hline HDL (mmol/l) & 9 & 367 & 0.04 & $-0.05,0.13$ & 0.35 & $81 \%$ & 0.61 \\
\hline $\mathrm{TG}(\mathrm{mmol} / \mathrm{l})$ & 9 & 367 & 0.02 & $-0.11,0.15$ & 0.77 & $40 \%$ & 0.56 \\
\hline DBP (mmHg) & 8 & 342 & 1.03 & $-0.86,2.92$ & 0.29 & $38 \%$ & 0.02 \\
\hline $\mathrm{SBP}(\mathrm{mmHg})$ & 8 & 342 & -2.97 & $-7.72,1.79$ & 0.22 & $60 \%$ & 0.64 \\
\hline BW (kg) & 7 & 410 & -0.32 & $-0.78,0.14$ & 0.17 & $2 \%$ & 0.65 \\
\hline \multicolumn{8}{|l|}{ CT vs AET } \\
\hline $\mathrm{HbA}_{1 \mathrm{c}}(\%)$ & 9 & 493 & -0.17 & $-0.31,-0.03$ & 0.02 & $21 \%$ & 0.17 \\
\hline $\mathrm{HbA}_{1 \mathrm{c}}(\mathrm{mmol} / \mathrm{mol})$ & & & -1.87 & $-3.41,-0.33$ & & & \\
\hline $\mathrm{FG}(\mathrm{mmol} / \mathrm{l})$ & 4 & 132 & -0.59 & $-1.25,0.08$ & 0.08 & $49 \%$ & 0.87 \\
\hline $\mathrm{TC}(\mathrm{mmol} / \mathrm{l})$ & 6 & 219 & -0.15 & $-0.35,0.05$ & 0.15 & $5 \%$ & 0.13 \\
\hline LDL (mmol/l) & 6 & 291 & -0.06 & $-0.24,0.13$ & 0.54 & $5 \%$ & 0.50 \\
\hline HDL (mmol/l) & 7 & 326 & 0.03 & $-0.03,0.09$ & 0.31 & $0 \%$ & 0.94 \\
\hline $\mathrm{TG}(\mathrm{mmol} / \mathrm{l})$ & 6 & 281 & -0.16 & $-0.35,0.03$ & 0.10 & $0 \%$ & 0.42 \\
\hline DBP $(\mathrm{mmHg})$ & 6 & 291 & -1.83 & $-4.67,1.01$ & 0.21 & $51 \%$ & 0.57 \\
\hline $\mathrm{SBP}(\mathrm{mmHg})$ & 6 & 291 & -0.81 & $-4.22,2.61$ & 0.64 & $0 \%$ & 0.09 \\
\hline BW (kg) & 8 & 450 & -0.95 & $-1.93,0.02$ & 0.05 & $42 \%$ & 0.97 \\
\hline \multicolumn{8}{|l|}{ CT vs RT } \\
\hline $\mathrm{HbA}_{1 \mathrm{c}}(\%)$ & 5 & 362 & -0.62 & $-0.95,-0.30$ & 0.0002 & $74 \%$ & 0.69 \\
\hline $\mathrm{HbA}_{1 \mathrm{c}}(\mathrm{mmol} / \mathrm{mol})$ & & & -6.82 & $-10.45,-3.3$ & & & \\
\hline $\mathrm{FG}(\mathrm{mmol} / \mathrm{l})$ & 3 & 99 & -1.99 & $-3.07,-0.90$ & 0.0003 & $61 \%$ & 0.45 \\
\hline $\mathrm{TC}(\mathrm{mmol} / \mathrm{l})$ & 3 & 99 & -0.12 & $-0.90,0.65$ & 0.75 & $84 \%$ & 0.72 \\
\hline LDL (mmol/l) & 4 & 218 & -0.14 & $-0.68,0.39$ & 0.59 & $83 \%$ & 0.74 \\
\hline HDL (mmol/l) & 4 & 218 & 0.10 & $-0.11,0.32$ & 0.33 & $91 \%$ & 0.48 \\
\hline TG (mmol/l) & 4 & 213 & -0.28 & $-0.46,-0.10$ & 0.003 & $0 \%$ & 0.31 \\
\hline DBP (mmHg) & 4 & 213 & -1.13 & $-3.55,1.29$ & 0.36 & $0 \%$ & 0.50 \\
\hline SBP (mmHg) & 4 & 206 & -4.42 & $-8.62,-0.21$ & 0.04 & $41 \%$ & 0.08 \\
\hline BW (kg) & 4 & 317 & -1.04 & $-2.07,-0.00$ & 0.05 & $0 \%$ & 0.03 \\
\hline
\end{tabular}

${ }^{a}$ Fixed effect meta-analysis if $I^{2} \leq 50 \%$

LDL, HDL and TG between AET and RT. However, AET resulted in a significant reduction in FG (MD $-0.90 \mathrm{mmol} / \mathrm{l}$; $95 \%$ CI $-1.71,-0.09 ; p=0.03, I^{2}=72 \%, 8$ trials, 245 participants) compared with RT (ESM Fig. 7). Compared with RT, $\mathrm{CT}$ resulted in a more pronounced decrease in FG (MD $-1.99 \mathrm{mmol} / \mathrm{l} ; 95 \% \mathrm{CI}-3.07,-0.90 ; p=0.0003, I^{2}=61 \%, 3$ trials, 99 participants) (ESM Fig. 8), TG (MD $-0.28 \mathrm{mmol} / \mathrm{l}$; $95 \%$ CI $-0.46,-0.10 ; p=0.003, I^{2}=0 \%, 4$ trials, 213 participants) (ESM Fig. 9) and SBP (MD $-4.42 \mathrm{mmHg}$; $95 \% \mathrm{CI}$ $-8.62,-0.21 ; p=0.04, I^{2}=41 \%, 4$ trials, 206 participants) (ESM Fig. 10).
Network meta-analysis

ESM Fig. 3 shows the network of the included trials. The pooled estimates of effect size for the comparison of AET vs RT vs CT using both direct and indirect evidence on glycaemic control and cardiovascular risk outcomes are summarised in ESM Table 3 (a fixed effect network meta-analysis for BW and TG, since $I^{2} \leq 50 \%$ ). For each outcome, a common between-study heterogeneity variable was assumed to reflect the variability between studies of all the interventions (ESM Table 3). The ranking 
probabilities of AET, RT and CT for each outcome are presented in ESM Table 4.

Both AET and CT were significantly more effective in reducing $\mathrm{HbA}_{1 \mathrm{c}}$ when compared with $\mathrm{RT}$. As shown in ESM Table 4, CT turned out to be the most effective exercise intervention with respect to reducing $\mathrm{HbA}_{1 \mathrm{c}}, \mathrm{FG}, \mathrm{TC}, \mathrm{LDL}$, TG, DBP, SBP and BW, and increasing HDL. CT resulted in a high $(>75 \%)$ probability of being best for most outcomes. There is greater uncertainty regarding which treatment is the best for LDL- and TC, although again CT yielded the highest probability of being best.

No evidence of inconsistency was found with Bayesian $p$ values for the difference between direct and indirect evidence all greater than 0.90 .

\section{Risk of bias}

The dropout rates ranged from $0 \%$ to $31 \%$, with five studies reporting dropout rates $<10 \%$ (ESM Table 1). Seven trials reported random sequence generation [16, 31, 32, 36-39], and only five trials reported allocation concealment [16, 31, $32,37,38]$. None of the studies reported the blinding of volunteers towards the mode of intervention (ESM Fig. 1). Eight trials performed intention-to-treat analysis [15-17, 32, 35, 37-39], and six trials appear to have had adequate blinding of the outcome assessment [16, 17, 31, 32, 37, 38]. High risk of bias was defined as fewer than four out of a maximum yield of six low risk of bias items using the risk of bias assessment tool from the Cochrane Collaboration (ESM Fig. 1). Seven high risk of bias trials (nine reports) were identified [15, 19,
20, 33-36, 39, 40], and sensitivity analyses were performed for studies with a high vs low risk of bias.

\section{Subgroup analysis/sensitivity analysis}

Subgroup analyses were performed comparing shortterm $(<6$ months) vs long-term ( $\geq 6$ months) trials (ESM Figs 11-13), obese (BMI $\geq 30 \mathrm{~kg} / \mathrm{m}^{2}$ ) vs non-obese $\left(\mathrm{BMI}<30 \mathrm{~kg} / \mathrm{m}^{2}\right.$ ) participants (ESM Figs 14-16) and sample size $(\geq 50$ vs $<50)$ (ESM Fig. 17-19). Overall, pooling the long-term trials resulted in significantly greater reductions of $\mathrm{HbA}_{1 \mathrm{c}}$ compared with short-term trials for all comparison groups. Furthermore, including only obese patients resulted in significant reductions in $\mathrm{HbA}_{1 \mathrm{c}}$. A smaller vs bigger sample size showed non-significant differences for $\mathrm{HbA}_{1 \mathrm{c}}$ when comparing AET vs RT. In contrast, comparisons for CT yielded significantly higher reductions in trials with a bigger sample size when compared with either AET or RT. Subgroup analysis comparing different measurement time points for $\mathrm{HbA}_{1 \mathrm{c}}$ provided no additional information (ESM Figs 20-22). Sensitivity analyses excluding trials with a high risk of bias changed the summary estimates and became statistically nonsignificant (Table 3).

\section{Publication bias}

Begg's and Egger's regression tests provided no evidence of a substantial publication bias. Funnel plots were generated only if specific outcome measures were provided by at least ten different trials. The plot with respect to change in effect size

Table 3 Low risk of bias sensitivity analysis (pairwise fixed/random effect meta-analysis) of effect size (95\% CIs) expressed as weighted MD for the effects of AET vs RT, CT vs AET and CT vs RT on glycaemic control, blood lipids, blood pressure and BW

\begin{tabular}{|c|c|c|c|c|c|c|}
\hline Outcomes & No. of studies & Sample size & MD & $95 \% \mathrm{CI}$ & $p$ values & Inconsistency $I^{2}$ \\
\hline \multicolumn{7}{|l|}{ AET vs RT } \\
\hline $\mathrm{HbA}_{1 \mathrm{c}}(\%)$ & 5 & 371 & -0.07 & $-0.22,0.08$ & 0.38 & $0 \%$ \\
\hline $\mathrm{HbA}_{1 \mathrm{c}}(\mathrm{mmol} / \mathrm{mol})$ & & & -0.77 & $-2.42,-0.88$ & & \\
\hline $\mathrm{FG}(\mathrm{mmol} / \mathrm{l})$ & 3 & 101 & -0.12 & $-0.68,0.44$ & 0.68 & $0 \%$ \\
\hline $\mathrm{TC}(\mathrm{mmol} / \mathrm{l})$ & 3 & 121 & 0.11 & $-0.12,0.33$ & 0.35 & $0 \%$ \\
\hline LDL (mmol/l) & 4 & 226 & -0.06 & $-0.20,0.09$ & 0.34 & $0 \%$ \\
\hline HDL (mmol/l) & 4 & 226 & 0.00 & $-0.09,0.09$ & 0.96 & $68 \%$ \\
\hline $\mathrm{TG}(\mathrm{mmol} / \mathrm{l})$ & 4 & 226 & 0.08 & $-0.09,0.26$ & 0.70 & $0 \%$ \\
\hline DBP (mmHg) & 4 & 226 & -0.86 & $-3.62,1.89$ & 0.54 & $0 \%$ \\
\hline $\mathrm{SBP}(\mathrm{mmHg})$ & 4 & 226 & -1.18 & $-7.53,5.17$ & 0.72 & $60 \%$ \\
\hline BW (kg) & 3 & 310 & -0.26 & $-0.82,0.29$ & 0.36 & $0 \%$ \\
\hline \multicolumn{7}{|l|}{ CT vs AET } \\
\hline $\mathrm{HbA}_{1 \mathrm{c}}(\%)$ & 4 & 332 & -0.10 & $-0.32,0.11$ & 0.35 & $0 \%$ \\
\hline $\mathrm{HbA}_{1 \mathrm{c}}(\mathrm{mmol} / \mathrm{mol})$ & & & -1.1 & $-3.52,-1.21$ & & \\
\hline HDL (mmol/l) & 3 & 184 & 0.07 & $-0.02,0.15$ & 0.12 & $34 \%$ \\
\hline $\mathrm{TG}(\mathrm{mmol} / \mathrm{l})$ & 3 & 184 & -0.28 & $-0.62,0.07$ & 0.11 & $19 \%$ \\
\hline BW (kg) & 4 & 332 & -1.80 & $-7.86,4.27$ & 0.56 & $64 \%$ \\
\hline
\end{tabular}


for $\mathrm{HbA}_{1 \mathrm{c}}$ in response to AET vs RT indicates little asymmetry. Thus, publication bias cannot be completely excluded as a factor affecting the results of the present meta-analysis (ESM Fig. 23).

\section{Discussion}

According to our literature search, this is the first network meta-analysis comparing the pooled effects of AET, RT and CT on glycaemic control, blood pressure and blood lipids in patients with type 2 diabetes. The results of the present metaanalyses showed that, in patients with established diabetes, AET might be more effective in reducing $\mathrm{HbA}_{1 \mathrm{c}}$ and $\mathrm{FG}$ when compared with RT. CT was more powerful in reducing $\mathrm{HbA}_{1 \mathrm{c}}$ compared with AET, and more effective in reducing $\mathrm{HbA}_{1 \mathrm{c}}$, FG and TG when compared with RT. However, these results could not be confirmed when only low risk of bias studies were included. Pooling both direct and indirect evidence on AET, RT and CT via network meta-analysis demonstrated that $\mathrm{CT}$ was the most efficacious exercise intervention regarding its impact on $\mathrm{HbA}_{1 \mathrm{c}}$, FG, HDL, TG, DBP and BW (with the respective probabilities of being ranked best following Bayesian network meta-analysis of $94 \%, 94 \%, 78 \%, 99 \%$, $84 \%$ and $97 \%$ ).

$\mathrm{HbA}_{1 \mathrm{c}}$ is not unanimously regarded to be a valid predictor of cardiovascular disease, thereby limiting the relevance of the present findings with respect to their clinical implications. The interpretation of the present data is further restricted by the fact that none of the studies evaluated the impact of their interventions on clinical outcomes. Data from epidemiological studies suggest that greater physical activity is associated with a reduced risk of all-cause mortality, mortality from cardiovascular disease and risk of type 2 diabetes [44-47].

A recent meta-analysis comparing RT with AET concluded that there is no evidence that RT differs from AET in its impact on cardiovascular risk factors and safety [9]. However, for some risk factors, the ranking probabilities of the Bayesian network meta-analysis suggest that AET was the second best exercise modality. However, these probabilities should not be overinterpreted, particularly since they are not very large (not close to 80-90\%). A dose-response meta-regression analysis by Umpierre et al [48] summarised the effects of CT, AET and RT on glycaemic control in patients with type 2 diabetes and concluded that the reduction in $\mathrm{HbA}_{1 \mathrm{c}}$ was associated with exercise frequency in supervised AET, and with the weekly volume of RT in supervised CT. Regarding the optimal dose, the authors speculated that there should be a minimal amount of AET (33 min per session) to elicit the effects of highvolume RT in CT [11].

The results of this meta-analysis are in line with data published by Chudyk et al [6] comparing CT, AET and RT with control groups. The authors concluded that RT is not significantly related to changes in $\mathrm{HbA}_{1 \mathrm{c}}$ levels or to changes in SBP in patients with type 2 diabetes if it is not combined with other forms of exercise. Moreover, splitting AET and RT sessions between different days might have additional benefits for glycaemic control [49]. This indicates that more elaborated training programmes might be of relevance.

According to a mechanistic model linking the combination of AET and RT with the improvement in glycaemic control in type 2 diabetes, RT enhances insulin sensitivity [50] via an increase in glucose transporter (GLUT)-4 content and an amplification of insulin signalling in muscle [51]. Similarly, AET increased GLUT-4 expression in the adipose tissue and skeletal muscle of patients with type 2 diabetes; however, the benefit of this adaption appears to be dependent on optimal beta cell function [52].

One strength of this systematic review is the application of a network meta-analysis. Direct and indirect evidence was used, taking into account the fact that AET, RT and CT were compared simultaneously in some studies. However, the multiple use of data from three-arm trials will lead to an overestimation of the corresponding data and should be avoided. The relevance of the present data is further emphasised by the small estimated between-studies heterogeneity variables as well as by the fact that there was no evidence of inconsistencies.

On the other hand, this systematic review has several limitations that should be taken into account when interpreting its findings. There is evidence that supervised exercise is more effective than unsupervised training [11], but in practice it seems unlikely that most patients would have access to supervised exercise regimens of this intensity. It is possible that either AET, RT or CT may be easier to perform effectively without supervision, thus affecting the external validity of these results since only studies with supervised training were included.

Although the network meta-analysis included all individuals for each outcome, the sample size of volunteers might be considered low when compared with drug trials. Several potential risk of bias characteristics were identified in the 14 included trials ( 7 trials described random sequence generation, 5 trials performed allocation concealment, 8 trials performed intention-to-treat analysis, and 6 trials had adequate blinding of outcome assessment). Taken together, more than $50 \%$ of the included trials were judged as being at high risk of bias. Therefore, the results of the present meta-analyses should be interpreted in a conservative manner.

There were some heterogeneities in study design especially with respect to the population characteristics (e.g. duration of type 2 diabetes, study length, BMI, age and ratio of male to female participants). Subgroup analyses showed that longterm trials as well as trials including obese participants with type 2 diabetes resulted in more pronounced beneficial effects 
on $\mathrm{HbA}_{1 \mathrm{c}}$, which might be due to carrying forward $\mathrm{HbA}_{1 \mathrm{c}}$ values under conditions of high dropout rates. However, no significant differences could be observed following a comparison of the dropout rates between short- and long-term studies in the present network meta-analysis. Another confounder might be the variations in the volume of exercise (min per week) prescribed. One study reported exercise duration (min per session) in the CT group to be twice as high as with their respective RT and/or AET counterparts [16]. However, a sensitivity analysis excluding this trial was able to confirm the results of the primary analysis.

With respect to the potential side effects (ESM Table 1), eight trials in the present meta-analysis reported adverse events such as hypoglycaemia, back pain, shoulder pain, musculoskeletal injury, tendonitis and other musculoskeletal discomforts following exercise, with no significant differences between the intervention groups. However, it remains possible that the number of adverse events will increase with the duration and intensity of exercise.

This systematic review and meta-analysis focused on randomised controlled trials comparing AET, RT and CT. Compared with AET or RT, CT interventions resulted in significantly more pronounced improvements in variables related to glycaemic control. With respect to single types of exercise intervention, AET was more effective in reducing $\mathrm{HbA}_{1 \mathrm{c}}$ and FG when compared with RT. However, the interpretation of these findings with respect to their clinical relevance is limited by the overall low to moderate quality of the studies included, the lack of information on clinically important outcomes, and the limited information on the adverse effects of exercise.

Funding This research received no specific grant from any funding agency in the public, commercial or not-for-profit sectors.

Duality of interest The authors declare that there is no duality of interest associated with this manuscript.

Contribution statement LS and GH conceived this study, LS, BM and SD analysed the data, and LS, BM, JK and GH contributed to the collection of data. All authors critically reviewed various drafts of the manuscript, and all authors approved the final version. GH is responsible for the integrity of the work as a whole.

\section{References}

1. Tuomilehto J, Lindstrom J, Eriksson JG et al (2001) Prevention of type 2 diabetes mellitus by changes in lifestyle among subjects with impaired glucose tolerance. N Engl J Med 344:1343-1350

2. Ajala O, English P, Pinkney J (2013) Systematic review and metaanalysis of different dietary approaches to the management of type 2 diabetes. Am J Clin Nutr 97:505-516

3. Schwingshackl L, Hoffmann G (2014) Comparison of the long-term effects of high-fat $v$. low-fat diet consumption on cardiometabolic risk factors in subjects with abnormal glucose metabolism: a systematic review and meta-analysis. Br J Nutr 111:2047-2058

4. Colberg SR, Sigal RJ, Fernhall B et al (2010) Exercise and type 2 diabetes: the American College of Sports Medicine and the American Diabetes Association: joint position statement. Diabetes Care 33:e147-e167

5. Snowling NJ, Hopkins WG (2006) Effects of different modes of exercise training on glucose control and risk factors for complications in type 2 diabetic patients: a meta-analysis. Diabetes Care 29:25182527

6. Chudyk A, Petrella RJ (2011) Effects of exercise on cardiovascular risk factors in type 2 diabetes: a meta-analysis. Diabetes Care 34: 1228-1237

7. Strasser B, Siebert U, Schobersberger W (2010) Resistance training in the treatment of the metabolic syndrome: a systematic review and meta-analysis of the effect of resistance training on metabolic clustering in patients with abnormal glucose metabolism. Sports Med 40: 397-415

8. Kelley GA, Kelley KS (2000) Progressive resistance exercise and resting blood pressure: a meta-analysis of randomized controlled trials. Hypertension 35:838-843

9. Yang Z, Scott CA, Mao C, Tang J, Farmer AJ (2013) Resistance exercise versus aerobic exercise for type 2 diabetes: a systematic review and meta-analysis. Sports Med 44:487-499

10. Schwingshackl L, Dias S, Strasser B, Hoffmann G (2013) Impact of different training modalities on anthropometric and metabolic characteristics in overweight/obese subjects: a systematic review and network meta-analysis. PLoS One 8:e82853

11. Umpierre D, Ribeiro PA, Kramer CK et al (2011) Physical activity advice only or structured exercise training and association with HbAlc levels in type 2 diabetes: a systematic review and metaanalysis. JAMA 305:1790-1799

12. Moher D, Liberati A, Tetzlaff J, Altman DG (2009) Preferred reporting items for systematic reviews and meta-analyses: the PRISMA statement. PLoS Med 6:e1000097

13. Higgins JP, Green S (eds) (2011) Cochrane handbook for systematic reviews on interventions 5.1.0 [updated March 2011] The Cochrane Collaboration. Available from www.cochrane-handbook.org

14. Higgins JP, Altman DG, Gotzsche PC et al (2011) The Cochrane Collaboration's tool for assessing risk of bias in randomised trials. BMJ 343:d5928

15. Jorge ML, de Oliveira VN, Resende NM et al (2011) The effects of aerobic, resistance, and combined exercise on metabolic control, inflammatory markers, adipocytokines, and muscle insulin signaling in patients with type 2 diabetes mellitus. Metabolism 60:1244-1252

16. Sigal RJ, Kenny GP, Boule NG et al (2007) Effects of aerobic training, resistance training, or both on glycemic control in type 2 diabetes - a randomized trial. Ann Intern Med 147:357-369

17. Balducci S, Zanuso S, Nicolucci A et al (2010) Anti-inflammatory effect of exercise training in subjects with type 2 diabetes and the metabolic syndrome is dependent on exercise modalities and independent of weight loss. Nutr Metab Cardiovasc Dis 20: $608-617$

18. Lambers $S$, van Laethem $C$, van Acker K, Calders P (2008) Influence of combined exercise training on indices of obesity, diabetes and cardiovascular risk in type 2 diabetes patients. Clin Rehabil 22:483492

19. Gram B, Christensen R, Christiansen C, Gram J (2010) Effects of nordic walking and exercise in type 2 diabetes mellitus: a randomized controlled trial. Clin J Sport Med 20:355-361

20. de Oliveira VN, Bessa A, Jorge ML et al (2012) The effect of different training programs on antioxidant status, oxidative stress, and metabolic control in type 2 diabetes. Appl Physiol Nutr Metab 37:334-344

21. Higgins JP, Thompson SG, Deeks JJ, Altman DG (2003) Measuring inconsistency in meta-analyses. BMJ 327:557-560 
22. Begg CB, Mazumdar M (1994) Operating characteristics of a rank correlation test for publication bias. Biometrics 50:1088-1101

23. Egger M, Davey Smith G, Schneider M, Minder C (1997) Bias in meta-analysis detected by a simple, graphical test. BMJ 315:629-634

24. Dias S, Sutton AJ, Ades AE, Welton NJ (2013) Evidence synthesis for decision making 2: a generalized linear modeling framework for pairwise and network meta-analysis of randomized controlled trials. Med Decis Making 33:607-617

25. Spiegelhalter DJ, Best NG, Carlin BP, van der Linde A (2002) Bayesian measures of model complexity and fit. J R Stat Soc (B) 64:583-616

26. Lunn DJ, Thomas A, Best N, Spiegelhalter D (2000) WinBUGS - a Bayesian modelling framework: concepts, structure, and extensibility. Stat Comput 10:325-337

27. Dias S, Welton NJ, Sutton AJ, Ades AE (2011) NICE DSU Technical Support Document 2: A Generalised Linear Modelling Framework for Pairwise and Network Meta-Analysis of Randomised Controlled Trials. Available from http://www.nicedsu.org.uk. Accessed 05 Sept 2013

28. Brooks SP, Gelman A (1998) Alternative methods for monitoring convergence of iterative simulations. J Comput Graph Stat 7:434455

29. Dias S, Welton NJ, Caldwell DM, Ades AE (2010) Checking consistency in mixed treatment comparison meta-analysis. Stat Med 29: 932-944

30. Dias S, Welton NJ, Sutton AJ et al (2013) Evidence synthesis for decision making 4: inconsistency in networks of evidence based on randomized controlled trials. Med Decis Making 33:641-656

31. Bacchi E, Negri C, Zanolin ME et al (2012) Metabolic effects of aerobic training and resistance training in type 2 diabetic subjects: a randomized controlled trial (the RAED2 study). Diabetes Care 35: 676-682

32. Church TS, Blair SN, Cocreham S et al (2010) Effects of aerobic and resistance training on hemoglobin Alc levels in patients with type 2 diabetes: a randomized controlled trial. JAMA 304:2253-2262

33. Cuff DJ, Meneilly GS, Martin A et al (2003) Effective exercise modality to reduce insulin resistance in women with type 2 diabetes. Diabetes Care 26:2977-2982

34. Kwon HR, Min KW, Ahn HJ et al (2011) Effects of aerobic exercise vs resistance training on endothelial function in women with type 2 diabetes mellitus. Diabetes Metab J 35:364-373

35. Ku YH, Han KA, Ahn H et al (2010) Resistance exercise did not alter intramuscular adipose tissue but reduced retinol-binding protein- 4 concentration in individuals with type 2 diabetes mellitus. J Int Med Res 38:782-791

36. Kadoglou NP, Fotiadis G, Kapelouzou A et al (2013) The differential anti-inflammatory effects of exercise modalities and their association with early carotid atherosclerosis progression in patients with type 2 diabetes. Diabet Med 30:e41-e50

37. Moe B, Augestad LB, Åsvold BO, Flanders WD (2011) Effects of aerobic versus resistance training on glycaemic control in men with type 2 diabetes. Eur J Sport Sci 11:365-374
38. Ng C, Goh S, Malhotra R, Ostbye T, Tai S (2010) Minimal difference between aerobic and progressive resistance exercise on metabolic profile and fitness in older adults with diabetes mellitus: a randomised trial. J Physiother 56:163-170

39. Sukala WR, Page R, Rowlands DS et al (2012) South Pacific Islanders resist type 2 diabetes: comparison of aerobic and resistance training. Eur J Appl Physiol 112:317-325

40. Yavari A, Najafipoor F, Aliasgharzadeh A et al (2012) Effect of aerobic exercise, resistance training or combined training on glycaemic control and cardiovascular risk factors in patients with type 2 diabetes. Biol Sport 29:135-143

41. Marcus RL, Smith S, Morrell G et al (2008) Comparison of combined aerobic and high-force eccentric resistance exercise with aerobic exercise only for people with type 2 diabetes mellitus. Phys Ther 88:1345-1354

42. Cauza E, Hanusch-Enserer U, Strasser B et al (2005) The relative benefits of endurance and strength training on the metabolic factors and muscle function of people with type 2 diabetes mellitus. Arch Phys Med Rehabil 86:1527-1533

43. Shenoy S, Arora E, Jaspal S (2009) Effects of progressive resistance training and aerobic exercise on type 2 diabetics in Indian population. Int J Diabetes Metab 17:27-30

44. Grontved A, Pan A, Mekary RA et al (2014) Muscle-strengthening and conditioning activities and risk of type 2 diabetes: a prospective study in two cohorts of US women. PLoS Med 11:e1001587

45. Grontved A, Rimm EB, Willett WC, Andersen LB, Hu FB (2012) A prospective study of weight training and risk of type 2 diabetes mellitus in men. Arch Intern Med 172:1306-1312

46. Li J, Siegrist J (2012) Physical activity and risk of cardiovascular disease-a meta-analysis of prospective cohort studies. Int J Environ Res Publ Health 9:391-407

47. Kokkinos P, Myers J, Nylen E et al (2009) Exercise capacity and all-cause mortality in African American and Caucasian men with type 2 diabetes. Diabetes Care 32:623-628

48. Umpierre D, Ribeiro PA, Schaan BD, Ribeiro JP (2013) Volume of supervised exercise training impacts glycaemic control in patients with type 2 diabetes: a systematic review with meta-regression analysis. Diabetologia 56:242-251

49. Oliveira C, Simoes M, Carvalho J, Ribeiro J (2012) Combined exercise for people with type 2 diabetes mellitus: a systematic review. Diabetes Res Clin Pract 98:187-198

50. Misra A, Alappan NK, Vikram NK et al (2008) Effect of supervised progressive resistance-exercise training protocol on insulin sensitivity, glycemia, lipids, and body composition in Asian Indians with type 2 diabetes. Diabetes Care 31:1282-1287

51. Tabata I, Suzuki Y, Fukunaga T et al (1985) (1999) Resistance training affects GLUT-4 content in skeletal muscle of humans after 19 days of head-down bed rest. J Appl Physiol 86:909914

52. Hussey SE, McGee SL, Garnham A et al (2011) Exercise training increases adipose tissue GLUT4 expression in patients with type 2 diabetes. Diabetes Obes Metab 13:959-962 